\title{
Structure \& dynamics of fractal-like particles made by agglomeration and sintering
}

Maximilian L. Eggersdorfer ${ }^{l}$ and Eirini Goudeli ${ }^{2, *}$

1. currently at Novartis Pharma AG, Basel, Switzerland.

2. Laboratory of Aerosol and Particle Technology, Department of Chemical Engineering, The University of Melbourne, Melbourne VIC, Australia.

Submitted to:

AIChE Journal

June 23, 2020

*Corresponding author: eirini.goudeli@unimelb.edu.au 


\begin{abstract}
The growth of silica nanoparticles by agglomeration and viscous flow sintering is studied from free molecular to transition regime by off-lattice even-driven (ED) simulations. Coagulation by simultaneous agglomeration and sintering takes place at high temperature environments, where sintering and collision rates are comparable. The effect of temperature on aggregate mobility and gyration radii, particle morphology and collisional enhancement is elucidated. The ratio between the characteristic sintering time and characteristic collision time controls the particle size and structure, quantified by the mass fractal dimension. The aggregate morphology depends solely on the ratio of characteristic times and is insensitive to the process temperature. When sintering is negligible, the overall collision frequency is $90 \%$ larger than that predicted by the classic Fuchs collision kernel for monodisperse agglomerates, in agreement with experiments. The ED-obtained quasi-self-preserving size distributions are consistent with mobility size distributions measured in hot-wall reactors and flame sprays.
\end{abstract}

Keywords: aerosols, coagulation, sintering, discrete element method

\title{
1. Introduction
}

Gas-phase reactors provide a versatile, scalable waste-free synthesis route capable of producing nanoparticles at tons per hour worldwide. ${ }^{1}$ Most importantly, it allows for the manufacture of nanoparticles with fine-tuned size and 
structural characteristics that can be used routinely for diverse applications, such as bone replacements, ${ }^{2}$ dental fillers, ${ }^{3}$ photocatalysts ${ }^{4,5}$ and catalysts for environmental remediation, ${ }^{6}$ to name a few. As one example, flame-made luminescent $\mathrm{CeO}_{2}: \mathrm{Eu}^{3+}$ nanoparticles have demonstrated highly sensitive biosensing activity for $\mathrm{H}_{2} \mathrm{O}_{2},{ }^{7}$ whose presence is correlated with severe diseases. ${ }^{8}$ Aerosol synthesis processes, such as flame spray pyrolysis, hot wall reactors, and laser ablation, typically lead to the formation of fractal-like aggregates (chemically or sinter bonded spheres) or agglomerates (physically bonded spheres or aggregates). The degree of overlap among the constituent primary particles greatly affects the final product properties, such as their mechanical stability and electron transport. Tuning the primary particle size and the size of the sinter necks is key for the performance of catalysts and gas sensors. ${ }^{9}$ For instance, flame-made $\beta$ - $\mathrm{Bi}_{2} \mathrm{O}_{3}$ catalysts with high degree of sintering exhibit stable fractal structure and increased current density which improved their performance in $\mathrm{CO}_{2}$ electroreduction. ${ }^{10}$

One important design criterion for gas-phase (flame) reactors is the high temperature particle residence time, which determines nanoparticle growth by gas reactions, surface growth, sintering and coagulation. Typically, the chemistry is rapid so that nucleation can be neglected, and coagulation and sintering dominate the particle growth. ${ }^{11}$ Hence, understanding the dynamics of aggregate formation during coagulation and sintering is necessary, especially in the transition regime. Knowing the collision frequency and sintering time can assist in modeling the particle growth mechanisms accurately, ${ }^{12}$ which are reasonably well understood for spherical particles. However, $a$ priori assumptions on the particle structure and sintering rate are often used to calculate the particle collision frequency function, which is used to describe the evolving particle number concentration in monodisperse population balance equation (PBE) models. The radius of gyration is commonly used as the collision radius in collision kernel equations, ${ }^{13,14,15}$ even though they might differ even up to $21 \% .{ }^{16}$ Elaborate expressions of the collision radius have been developed by Monte Carlo (MC) simulations ${ }^{17,18}$ to improve the accuracy of the collision kernel. Discrete element method (DEM) simulations ${ }^{19,20,21}$ account for the velocity and detailed structural characteristics of individual aggregates or agglomerates and do not require a priori knowledge of a coagulation kernel. Recently, it was shown that agglomerates consisting of monodisperse primary particles in point contact attain their asymptotic fractal-like structure faster than those consisting of polydisperse primary particles, ${ }^{20}$ as the later exhibit larger collisional area that increases the coagulation rate. Nonetheless, once the agglomerates attain their asymptotic fractal dimension, $D_{f}$, and their self-preserving size distribution (SPSD) they coagulate with identical 
collision rates in the free molecular regime, regardless of primary particle polydispersity. This coagulation rate was $35 \%$ bigger than that of monodisperse agglomerates having $D_{f}=1.9 .{ }^{20}$

Furthermore, during particle growth in the gas-phase, the aggregate structure evolves from $D_{f}=3$, corresponding to spheres, down to $\sim 1.8$, corresponding to fractal-like aggregates made by cluster-cluster agglomeration. Artelt et al. ${ }^{22}$ proposed an empirical evolution of $D_{f}$ and showed the importance of accounting the evolving particle structure during early growth stages on the agglomerate collision frequency using PBE. The structural effect is significant in the free molecular regime where sintering and coagulation primarily take place at the high temperature region of aerosol reactors. Al Zaitone et al. ${ }^{23}$ simulated the growth of nanoparticle structures by simultaneous coagulation, sintering and surface growth. Coagulation was modeled by a MC algorithm and sintering by the successive overlap of spheres along a backbone in an aggregate. An empirical relation between characteristic times and $D_{f}$ was developed. Sander et al. ${ }^{24}$ used a stochastic particle model to describe the formation of $\mathrm{SiO}_{2}$, accounting for each contact between neighboring particles within an aggregate and the effect of polydispersity and fitted their results against experiments of Seto et al. ${ }^{25}$ Goudeli et al. ${ }^{15}$ employed a DEM-derived ${ }^{19} D_{f}$ equation for unsintered agglomerates consisting of monodisperse primary particles in a simple monodisperse PBE model, showing that neglecting the evolving particle morphology can underpredict the agglomerate size by up to $30 \%$. In the above models, however, the relation between structure, coagulation and sintering rates was not fully resolved. Furthermore, the effect of sintering on the collision frequency function of aggregates remains unknown.

Here, the evolving ratio of the collision and sintering rates is fully resolved during aerosol growth from small spherical nuclei until the formation of fractal-like aggregates. The evolution of the particle morphology is determined on-the-fly and the dynamics of aggregate growth are quantified without making a priori assumptions on the coagulation rate. The formation of amorphous silica nanoparticles by coagulation and sintering in the free molecular and transition regimes is simulated by extending prior event-driven (ED) simulations of coagulation by pure agglomeration ${ }^{19}$ to account for concurrent multiparticle sintering dynamics. Aggregate sintering is modeled by a viscous flow sintering mechanism accounting intrinsically for primary particle polydispersity and aggregate structure. The collision frequency enhancement and particle size distributions are extracted from the simulations for particles coagulation by agglomeration and sintering at various temperatures ranging from 1000 to $2000 \mathrm{~K}$. Silica, one of the largest aerosol commodities, is used as a model system for the present simulations. This approach provides detail at the single particle level and allows quantifying the effect of process temperature on the evolving 
particle structure, size distribution and coagulation rate for large populations of small aggregates that are either difficult to determine experimentally or require tedious measurements, such as transmission electron microscopy (TEM) counting or differential mobility analyzer measurements coupled with mass spectrometry. These variables are required as input in larger length and time scale models, such as sectional models or particle dynamics coupled with fluid dynamics simulations, and their accuracy is critical for the design of aerosol reactors at industrial scale.

\section{Theory}

\subsection{Agglomerate \& aggregate size, structure and collision frequency}

The agglomerate radius of gyration, $r_{g}$, and mobility radius, $r_{m}$, scale with the number of primary particles per agglomerate, $n_{p}$, by the following power laws: $:^{26,27}$

$$
n_{p}=k_{n}\left(\frac{r_{g}}{r_{p}}\right)^{D_{f}}=k_{f m}\left(\frac{r_{m}}{r_{p}}\right)^{D_{f i n}}
$$

where $r_{p}$ is the primary particle radius, $k_{n}$ and $k_{f m}$ are proportionality constants and the particle structure descriptors $D_{f}$ and $D_{f m}$ are the mass fractal dimension and mass-mobility exponent, respectively. The $r_{g}$ is calculated by the mean square displacement of the agglomerate mass from its center of mass and the $r_{m}$ is calculated based on the rotationally averaged projected area of the agglomerate ${ }^{28}$ For sintered aggregates consisting of truncated primary particles, the surface area mean radius, $r_{v a}=3 V / A$, where $V$ and $A$ are the aggregate volume and surface area, is used as an equivalent primary particle radius.

The dynamics of agglomerate formation are quantified by the overall collision frequency function. A widely used coagulation rate that is valid in the entire particle size spectrum region is the classic Fuchs interpolation formula, $\beta_{\text {Fuchs }}:{ }^{29}$

$$
\beta_{F u c h s}=\beta_{c o} \cdot\left[\frac{d_{i}+d_{j}}{d_{i}+d_{j}+2 \delta_{r}}+\frac{8\left(D_{i}+D_{j}\right)}{\bar{c}_{r}\left(d_{i}+d_{j}\right)}\right]^{-1}
$$

with

$$
\begin{gathered}
\delta_{r}=\sqrt{\delta_{1}^{2}+\delta_{2}^{2}} \text {, with } \\
\delta_{i}=\frac{1}{3 d_{p, i} \lambda_{a, i}}\left[\left(d_{p, i}+\lambda_{a, i}\right)^{3}-\left(d_{p, i}^{2}+\lambda_{a, i}^{2}\right)^{3 / 2}\right]-d_{p, i} \\
D_{i}=\frac{k_{B} T C_{S, i}}{3 \pi \mu d_{p}}
\end{gathered}
$$


and

$$
\begin{gathered}
\bar{c}_{r}=\sqrt{\bar{c}_{1}^{2}+\bar{c}_{2}^{2}}, \text { with } \\
\bar{c}_{i}=\sqrt{\frac{48 k_{B} T}{\pi^{2} \rho_{p} d_{p, i}^{3}}}
\end{gathered}
$$

where $\beta_{c o}$ is the collision frequency function in the continuum regime, $d_{i}$ and $d_{p}$ are the diameter of the colliding species and primary particles, respectively, $\delta_{i}$ and $\delta_{r}$ are the mean and average mean distance, respectively, $D$ is the diffusion coefficient, $C_{s}$ is the Cunningham slip correction factor, $\mu$ is the viscosity of the surrounding fluid, $\lambda_{\alpha}$ is the agglomerate stopping distance $\left(\lambda_{a, i}=\frac{8 D_{i}}{\pi \overline{c_{i}}}\right), \overline{c_{l}}$ and $\overline{c_{r}}$ are the mean and average mean particles velocity, and $k_{B}, T$ and $\rho_{p}$ are the Boltzmann constant, temperature and bulk particle density, respectively.

\subsection{Numerical Implementation}

Two thousand primary particles are randomly distributed in an initially cubic simulation domain with periodic boundary conditions as described by Goudeli et al. ${ }^{19}$ In the free molecular regime, each particle is assumed to be in thermal equilibrium with the surrounding gas, following a Maxwellian velocity distribution. ${ }^{30}$ The particles follow ballistic trajectories with velocity that depends only on the particle mass. ${ }^{31}$ In the transition regime, where the gas mean free path is comparable to the agglomerate radius, the particle trajectories are a blend of ballistic and Brownian motion. During coagulation, particle sintering is accounted for, while other interparticle forces, such as van der Waals, electric and hydrodynamic interactions are neglected along with particle rotations. Particles stick inelastically upon collision with a sticking probability of unity. This is a reasonably good assumption for flamemade (amorphous), small silica nanoclusters $(0.5-1 \mathrm{~nm})$ exhibiting sticking probability of 1 for impact velocities at least up to $1000 \mathrm{~m} / \mathrm{s}^{32}$, as well as for larger amorphous silica spheres with radii up to $15 \mathrm{~nm}^{33}$, as evidenced by molecular dynamics simulations. Here, at least $92 \%$ of the $0.5 \mathrm{~nm}$-initial primary particles travel with velocities lower than $1000 \mathrm{~m} / \mathrm{s}$.

The above assumption of a sticking probability of 1 , needs to be revisited depending on the particle size, material and the nature of inter-particle interactions. For example, rebound becomes significant when combustion generated carbonaceous nanoparticles are colliding, exhibiting collision efficiencies bellow 1, especially for primary particle radii smaller than $5 \mathrm{~nm}^{34}$. Furthermore, for big nano-sized amorphous primary particles $\left(r_{p}>15 \mathrm{~nm}\right)^{33,35}$ or for micron-sized granules with viscous binding liquid coatings ${ }^{36}$, rebound events are probable. Such rebound events 
can be accounted for in the present off-lattice ED model for example by introducing different restitution coefficients for various particle sizes.

Once a particle collides with another particle or once it or has traveled its persistence length, it changes direction. The trajectories of the newly formed particles are calculated by the momentum balance. The particle drag is quantified by using the Stokes friction coefficient with the Cunningham correction factor. The particle bombardment from the surrounding fluid molecules is quantified by a stochastic, fluctuating force in the transition regime, as described in Heine and Pratsinis ${ }^{37}$. Aggregates or agglomerates are formed by subsequent particleparticle, particle-cluster and cluster-cluster collisions. Cluster-cluster coagulation is dominant, especially at long residence times that single spherical particles have been consumed and mostly large aggregates are present.

The relaxation time for particles to adjust to a change in the flow velocity is: ${ }^{38}$

$$
\tau_{p}=\frac{2 r_{m} \rho_{p} C_{s}}{18 \mu}
$$

and is much shorter ${ }^{38}$ than the collision time, $\tau_{c}$, which is defined as $:^{39}$

$$
\tau_{c}=\frac{2}{\beta N}
$$

at isothermal conditions. The $\beta$ and $N$ in eq. 7 correspond to the collision frequency function and the number concentration, respectively, at every time step. The time between particle collisions is calculated with an eventdriven algorithm..$^{40}$ The primary particles are initially monodisperse with solid volume fraction of $\varphi_{\mathrm{s}}=10^{-4}$ in all simulations. The effective volume fraction, $\varphi_{e f f}$, increases as fractal-like aggregates of agglomerates are formed with void space in-between their branches. Here, particle growth is elucidated at dilute conditions, so $\varphi_{\text {eff }}$ remains below 0.01 at all simulations to avoid high concentration effects on the collision frequency function and particle size distribution. ${ }^{31,37}$ High concentrations can result in coagulation that proceeds much faster than the Smoluchowski coagulation and particles reach gelation very quickly. ${ }^{37,41}$

The degree of aggregation depends on the evolution of the ratio of the characteristic collision time, $\tau_{c}$, and the characteristic sintering time $\tau_{s}$. Various sintering rates have been proposed ${ }^{42,43,44}$ for amorphous silica nanoparticles based on viscous flow sintering mechanism due to low viscosity. For example, Kirchhof et al. ${ }^{44}$ derived experimentally the sintering rate of silica particles in high-temperature sintering flow reactors for primary particle diameters of $8-80 \mathrm{~nm}$ at temperatures of $1300-1600{ }^{\circ} \mathrm{C}$. Here, the sintering rate of Tsantilis et al. ${ }^{43}$, which is derived from Frenkel's theoretical equation on the viscous flow mechanism ${ }^{45}$, using the material properties of 
silica $^{42}$, is used:

$$
\tau_{s}=6.5 \cdot 10^{-13} d_{p} \exp \left(\frac{8.3 \cdot 10^{4}}{T}\left(1-\frac{1 \cdot 10^{-9}}{d_{p}}\right)\right)
$$

for the range of process temperature $(1000-2000 \mathrm{~K})$ and primary particle diameters $\left(d_{p}=1-10 \mathrm{~nm}\right)$ employed in ED simulations. Equation 8 accounts for an empirical correction for the effect of melting temperature on small particle sizes that sinter rapidly. The detailed derivation of eq. 8 is discussed in Tsantilis et al. ${ }^{43}$

Figure 1 shows the schematic representation of the various growth mechanisms taking place depending on the ratio of sintering rate, $\tau_{s}$, and the collision rate, $\tau_{c}$. The time between two collision events sets the simulation time step, $\Delta t_{c}$. For $\tau_{s}<<\tau_{c}$ (Fig. 1a), particles behave as liquid-like spheres and coalesce fully upon collision. In the next timestep, the two colliding particles are replaced by a larger sphere with the same mass as the sum of the masses of the coalescing species. The mechanism of full coalescence is significant at high temperature flame regions. Further downstream in flames, particles have grown upon consecutive collisions while temperature starts to drop. In those flame regions, the sintering and collision rates are comparable (Fig. 1b, $\tau_{s} \sim \tau_{c}$ ) and particles coalesce partially (sinter). During $\Delta t_{s}$, the colliding particles start forming necks between them that grow (based on eq. 8) until the sintered aggregate collides with another particle, forming a larger aggregate at $t+\Delta t_{c}$. The free accessible surface, volume and neck contract areas between the sintered particles (truncated spheres) are determined with Vorlume ${ }^{46}$. In the limit of pure agglomeration $\tau_{s} \ll<\tau_{c}$ (Fig. 1c), sintering is less significant and the particles form fractal-like agglomerates with their constituent primary particles in point contact. The particle growth at conditions where the limit of pure agglomeration is dominant (Fig. 1c), has been presented in Goudeli et al. ${ }^{19,20}$

\section{Figure 1}

The overall collision frequency function is calculated by the present ED simulations by: ${ }^{37}$

$$
\beta=2\left(\frac{1}{N(t)}-\frac{1}{N(t-\Delta t)}\right) / \Delta t
$$

where $N$ is the number concentration at timesteps $t$ and $t-\Delta t$, respectively. The detailed simulation parameters and material properties are shown in Table 1. More rigorous equations of the binary rate of colliding particles during granulation of microparticles have been proposed by Reinhold and Briesen ${ }^{47}$, that apply to constant particle size distributions. Here, eq. 9 is used to derive the integral collision kernel as it can be applied to the evolving particle size distribution of the simulated aggregate populations.

\section{Table 1}




\section{Results and Discussion}

Full coalescence $\left(\tau_{s}<<\tau_{c}\right)$ and coagulation by pure agglomeration $\left(\tau_{s}>>\tau_{c}\right)$ are limiting cases of particle growth in aerosol particle synthesis. Coagulation by full coalescence is dominant at high temperatures environments or for small particles sizes and is typically encountered at the early stages of particle formation in flames, plasmas, hotwall or laser reactors. For large particle sizes and low temperature regions, e.g. downstream in aerosol reactors with high cooling rates, coagulation by agglomeration prevails. Non-spherical fractal-like aggregates are formed at process conditions where both coagulation and sintering take place.

\subsection{Validation with Full Coalescence}

The present ED simulations are validated for coagulation of particles by full coalescence $\left(\tau_{s}<<\tau_{c}\right)$. For fully coalescing nanoparticles, perfect spheres are formed upon collision and a coagulation rate $\sim 18 \%$ higher than theoretical collision kernel for spherical particles in the free molecular regime under dilute conditions is obtained. ${ }^{48}$ In the transition regime, the ED-obtained collision rate follows closely the collision frequency of Fuchs ${ }^{29}$ (Supplementary Information: Fig. S2a). These spherical particles rapidly reach their SPSD (Supplementary Information: Fig. S2b) with number- and volume- based geometric standard deviations $\sigma_{g, n}=1.46$ and $\sigma_{g, v}=1.33$, respectively. ${ }^{49}$ The viscous flow sintering simulations have been validated in Eggersdorfer et al. ${ }^{50}$

\subsection{Coagulation - Agglomeration}

Figure 2 shows snapshots of exemplary aggregates growing by agglomeration and sintering at temperature, $T=2000$ $\mathrm{K}$, and initial primary particle radius, $r_{p}$, of $0.5,1,2$ and $5 \mathrm{~nm}$. The aggregates are shown at different stages of their growth, corresponding to increasing effective number of primary particles per aggregate, $n_{p}$. During coagulation spherical particles collide forming either bigger spherical nanoparticles or sintered aggregates increasing their radius of gyration, $r_{g}$, and mobility radius, $r_{m}$. The equivalent number of primary particles per aggregate with radius $r_{p, 0}$ also increases. When the initial size of primary particles is small (i.e., $r_{p, 0}=0.5 \mathrm{~nm}$, left column) sintering prevails leading to large primary particles among which big sinter necks are formed. As the initial $r_{p, 0}$ increases, sintering contributes less to the growth of primary particles and at sufficiently long times (or increased $n_{p}$ ) agglomeration 
takes over completely leading to branched, fractal-like agglomerates. For a given $n_{p}$ (e.g., 515), more compact structures are formed when $r_{p, 0}$ is small, while more fractal-like aggregates are formed when the constituent primary particles are large. For example, these structural differences are evidenced by the differences in the $r_{g}$ of aggregates with $r_{p, 0}=5 \mathrm{~nm}\left(r_{g}=119 \mathrm{~nm}\right)$ which is about 30 times larger than those consisting of primary particles with $r_{p, 0}=$ $0.5 \mathrm{~nm}\left(r_{g}=4.1 \mathrm{~nm}\right)$. The aggregate $r_{m}$ is $71.7 \mathrm{~nm}$ for $r_{p, 0}=5 \mathrm{~nm}$ while for small $r_{p, 0}(=0.5 \mathrm{~nm})$, the $r_{m}$ is comparable to $r_{g}$, due to increased particle compactness.

Figure 2

Figure 3 shows the evolution of the geometric mean radius of gyration, $r_{g}$ (broken lines), mobility radius, $r_{m}$ (solid lines), and primary particles (PP) radius, $r_{p}$ (dotted lines), as function of the effective number of PPs per aggregate/agglomerate, $n_{p}$, with initial PP radius, $r_{p, 0}=1 \mathrm{~nm}$, at (a) 1000, (b) 1500, (c) 1750 and (d) $2000 \mathrm{~K}$. The highlighted areas represent the onset of aggregation, where the formed aggregates are perfect spheres $\left(r_{m}=r_{p}\right)$ as sintering is more prevalent than agglomeration, i.e. the characteristic sintering time, $\tau_{s}$, is smaller than the characteristic coagulation time, $\tau_{c}\left(\tau_{s}<\tau_{c}\right)$. However, these characteristic times change continuously during particle growth as the primary particles increase in size.

At $T=1000 \mathrm{~K}$ (Fig. 3a), sintering practically does not take place and agglomeration is the most dominant growth mechanism $\left(\tau_{s}>\tau_{c}\right)$. Thus, the $r_{p}$ remains constant as the agglomerates grow upon collisions increasing only their $r_{g}$ and $r_{m}$. It should be noted here that, due to lack of sintering, all agglomerates consist of monodisperse primary particles (Fig. 3a: insets). As temperature increases, sintering becomes more dominant and the size of the constituent primary particles, $r_{p}$, increases with increasing $n_{p}$ (or time). At high temperatures, nanoparticle sintering becomes more dominant, resulting in continuous increase of the average $r_{p}$, which has not reached the final particle diameter for the conditions investigated here. At the early stages of particle growth, aggregates grow by particleparticle and particle-cluster collisions and fully coalesce up to a critical $n_{p}$ (Fig. $3 \mathrm{~b}-\mathrm{d}$ : diamonds) above which $r_{m}>$ $r_{p}$. The critical $n_{p}$ required to form non-spherical aggregates with $D_{f}<3$ (as discussed in Fig. 7) corresponds to about 4 primary particles at $T=1500 \mathrm{~K}$ and increases to about 10 and 30 when temperature increases to 1750 and $2000 \mathrm{~K}$, respectively.

\section{Figure 3}

Figure 4 shows the evolution of the number-based geometric standard deviation of the radius of gyration, $\sigma_{g, g}$ (broken lines), mobility radius, $\sigma_{g, m}$ (solid lines), and volume-equivalent radius, $\sigma_{g, v}$ (dotted lines), as function of 
the effective number of PPs per aggregate/agglomerate, $n_{p}$, with initial PP radius, $r_{p, 0}=1 \mathrm{~nm}$, at (a) 1000, (b) 1500 , (c) 1750 and (d) $2000 \mathrm{~K}$. The highlighted areas represent the region where $r_{m}=r_{p}$, similar to Fig. 3 . At $T=1000 \mathrm{~K}$, where particles grow solely by agglomeration, the geometric standard deviations are initially $\left(n_{p}=1\right)$ equal to 1 , corresponding to a population of monodisperse primary particles. As $n_{p}$ increases, the $\sigma_{g, g}, \sigma_{g, m}$ and $\sigma_{g, v}$ increase attaining their free molecular SPSD values of 2.27, 2.03 and 1.41 (horizontal lines), respectively, consistent with Goudeli et al. ${ }^{19}$ When large agglomerates with $n_{p}>300$ are formed, $\sigma_{g}$ decreases as particle growth takes place in the transition regime.

When sintering prevails (i.e., at higher reactor temperature), the attainment of the quasi-SPSD is delayed and narrower aggregate size distributions are observed. In fact, sintered aggregates exhibit geometric standard deviations that lie between the SPSD limits for pure agglomerates $\left(\sigma_{g, g}=2.27, \sigma_{g, m}=2.03\right.$ and $\left.\sigma_{g, v}=1.41\right)$ and perfect spheres $\left(\sigma_{g, n}=1.46^{49}\right.$ and $\sigma_{g, v}=1.33^{49}$, Supplementary Information: Fig. S2b) in the free molecular regime. Before the onset of aggregation $\left(\tau_{s}<\tau_{c}\right), \sigma_{g, m}$ is comparable to $\sigma_{g, v}$, and they only start to differentiate when bigger aggregates are formed. Once the coagulation rate becomes bigger than the sintering rate, the $\sigma_{g, m}$ increases exhibiting values below 1.75 , consistent with differential mobility analyzer experiments in flame sprays that revealed $\sigma_{g, m}=$ $1.45-1.58$, even for bigger aggregates. ${ }^{51}$ The quasi-SPSD $\sigma_{g}$ derived from ED simulations are also in agreement with the evolution of TEM-obtained $\sigma_{g, g}$ and $\sigma_{g, v}$ for small flame-made zirconia nanoparticles ${ }^{52}$ with the same volume-equivalent radius $\left(r_{v a}=5 \mathrm{~nm}\right)$ and radius of gyration (Supplementary Information: Fig. S3).

As the temperature increases and sintering is enhanced, both the $r_{g}$ - and the $r_{m}$-based quasi-SPSD narrow down, in agreement with small-angle X-ray scattering (SAXS) measurements in laminar diffusion flames ${ }^{53}$ and mobility measurements in flame spray pyrolysis reactors ${ }^{51}$ that showed narrower silica size distributions as the particles become more compact (the $D_{f}$ and $D_{f m}$ decreases). For example, at $T=2000 \mathrm{~K}$ (Fig. 4d), the quasi-selfpreserving $\sigma_{g, m}$ drops by $\sim 20 \%$ from the self-preserving $\sigma_{g, m}$ of 2.03 corresponding to pure agglomeration, as sintering suppresses particle growth. At all temperatures, however, $\sigma_{g, v}$, reaches its self-preserving value which is identical to the $\sigma_{g, v}$, of pure agglomeration in the absence of sintering. The average aggregate and primary particle size (Fig. 3) and their corresponding geometric standard deviations (Fig. 4) can be used to obtain the detailed particle size distributions from monodisperse models without solving population balances.

The ED simulations employed here can also capture the growth of larger flame-made particles. The $r_{g}$ and $r_{m}$ of silica aggregates consisting of primary particles with initial $r_{p, 0}=10 \mathrm{~nm}$, obtained by transmission electron 
microscopy (TEM) and scanning mobility particle sizing (SMPS) measurements, respectively, exhibit the same evolution with zirconia aggregates having BET radius of $\sim 10 \mathrm{~nm}$, formed by flame spray pyrolysis with $5 \mathrm{~mL} / \mathrm{min}$ precursor solution and $2 \mathrm{~L} / \mathrm{min}$ dispersion oxygen ${ }^{54}$ (Supplementary Information: Fig. S4a). In fact, the present ED can capture accurately the particle growth early on, where the temperature employed in the simulations is comparable to the flame temperature, but underestimate the aggregate size further downstream the flame, where temperature drops by more than $1000 \mathrm{~K}$ and coagulation prevails. Furthermore, the $\sigma_{g, g}$ of those flame-made zirconia aggregates is larger than their $\sigma_{g, m}$, consistent with the present results (Supplementary Information: Fig. S4b). The simulations are in better agreement with the experimental $r_{m}$ and $\sigma_{g, m}$ rather than with $r_{g}$ and $\sigma_{g, g}$, possibly due to the fact that the 3D nature of the fractal-like aggregates is not accurately quantified by 2D TEM image counting. Notably, the simulations are performed without any fitting to the experimental data but serve as a guidance for experiments.

\section{Figure 4}

Figure 5 shows the ED-derived mobility size distributions of silica aggregates with $r_{p, 0}=1 \mathrm{~nm}$ growing by simultaneous agglomeration and sintering for $5.8 \cdot 10^{-8}$ (squares), $1.2 \cdot 10^{-7}$ (triangles), $4 \cdot 10^{-7}$ (circles), $6 \cdot 10^{-7}$ (diamonds) and $3 \cdot 10^{-6} \mathrm{~s}$ (down triangles) at (a) 1000, (b) 1500, (c) 1750 and (d) $2000 \mathrm{~K}$. Representative aggregate structures are shown exemplarily at $6 \cdot 10^{-7} \mathrm{~s}$ for three different temperatures (insets). At $T=1000 \mathrm{~K}$, agglomeration is the dominant growth mechanism. The agglomerates attain quickly the SPSD in the free molecular and quasiSPSD in the transition regime, as shown by the evolution of the geometric standard deviations based on the radius of gyration, mobility radius and volume equivalent radius (Fig. 4a). As temperature increases, sintering prevails and suppresses the growth of fractal-like aggregates. Instead, more compact structures are formed, which exhibit narrower mobility size distributions that are shifted to smaller average mobility diameters.

\section{Figure 5}

Figure 6a shows the mobility size distributions of Fig. 5 normalized by the geometric mean mobility diameter, $\left\langle d_{m}\right\rangle$, for aggregates formed by initial primary particles having $r_{p, 0}=0.5$ (purple triangles), 1 (red circles) and $5 \mathrm{~nm}$ (blue diamonds) derived by ED simulations (symbols) at $1750 \mathrm{~K}$. The ED simulations are in agreement with mobility size measurements obtained in hot wall reactors (gray solid line ${ }^{25}$ ) and flame sprays (dot-broken orange line ${ }^{51}$ ). The volume-based geometric standard deviation, $\sigma_{g, v}$, is presented in Fig. $6 \mathrm{~b}$ as function of the gasbased Knudsen number, $K n=\lambda / r_{m}$, and the SPSD limits for the aggregates of Fig. 6 a having $r_{p, 0}=0.5$ (dotted purple 
line), 1 (dot-broken red line), 2 (broken green line) and $5 \mathrm{~nm}$ (solid blue line). The horizontal lines correspond to the $\sigma_{g, v}$ of the SPSD for fully coalescing nanoparticles and agglomerates in point-contact attaining a fractal dimension, $D_{f}$, of 1.9 .

Initially, the particles coalesce fully upon collision at $1750 \mathrm{~K}$, regardless of their initial primary particle size, as $\tau_{\mathrm{s}} \ll \tau_{c}$. Aggregates with small initial primary particle size (i.e., $0.5 \mathrm{~nm}$ ), reach the SPSD of full coalescence with $\sigma_{g, v}=1.33$ down to $K n \approx 200-400$. Then the distributions become broader approaching the SPSD limit for agglomeration $\left(\sigma_{g, v}=1.41\right)$ at $K n \approx 150$. Larger particles do not have sufficient time to fully coalesce between collisions and form small aggregates, as shown in Fig. 2. As such, their $\sigma_{g, v}$ exceeds the SPSD limit of full coalescence approaching the self-preserving limit of 1.41 for pure agglomeration in the free molecular regime. At longer simulation times (smaller $K n$ ), the $\sigma_{g, v}$ decreases as the aggregates approach the transition regime.

\section{Figure 6}

The aggregate structure affects various particle properties, such as their mechanical and optical properties as well as the overall collision frequency function. The $D_{f}$ is commonly used to quantify the morphology of aggregates and agglomerates, taking values of 3 for spheres and 1.9 for agglomerates made by ballistic clustercluster agglomeration. ${ }^{55}$ Here, the $D_{f}$ is determined by fitting eq. 1 to an ensemble of $1000-2000$ aggregates at each time step. Figure 7 a shows the evolution of the mass fractal dimension, $D_{f}$, of silica aggregates made by agglomeration and sintering of initially monodisperse primary particles with radius of 0.5 (solid line), 1 (broken line), 2 (dot-broken line) and $5 \mathrm{~nm}$ (dotted line) at $2000 \mathrm{~K}$ as function of the effective $n_{p}$. Initially, the simulation box contains only spherical primary particles, which results to an average $D_{f}$ of 3 for the entire simulated population. As these spheres with $D_{f}=3$ grow even more, they require longer times to coalesce, leading to compact but nonspherical aggregates with $D_{f}<3$, upon concurrent agglomeration with other nanoparticles. The particles with $r_{p, 0}=5$ $\mathrm{nm}$ are the first to form aggregates, as discussed in Fig. 2. The critical $n_{p}$ that indicates the onset of aggregation in Fig. 3 corresponds to the $n_{p}$ where $D_{f}$ first becomes smaller than 3, i.e. the first non-spherical particles are formed. At a given temperature, this critical number increases as the initial primary particle size increases, indicating that the formed aggregates maintain their spherical shape longer. The critical $n_{p}$ indicating the onset of aggregation is only 2 for $r_{p, 0}=5 \mathrm{~nm}$ but increases to 300 for constituent number of primary particles with $r_{p, 0}=0.5 \mathrm{~nm}$.

Figure $7 \mathrm{~b}$ shows the evolution of the exponents $D_{f}$ (thick lines) and $D_{f m}$ (thin lines) as function of the dimensionless characteristic time, $\tau_{s} / \tau_{c}$, of silica aggregates with $r_{p, 0}=1 \mathrm{~nm}$ at $T=1500$ (solid gray lines), 1750 
(broken green lines) and $2000 \mathrm{~K}$ (dot-broken red lines). The $\tau_{s} / \tau_{c}$ indicates the regions that sintering and agglomeration are more significant. The $\tau_{c}$ is determined by eq. 7 using the ED-derived $\beta$ (eq. 9) and $N$, while $\tau_{s}$ is obtained by the sintering rate of Tsantilis et al. ${ }^{43}$ (eq. 8) using the geometric mean primary particle radius at each time step. The simulated populations of aggregates maintain an average $D_{f}$ of 3 for $\tau_{s} / \tau_{c}<1$ and follow the same evolutions with the dimensionless characteristic time independent of temperature. Therefore, only the ratio $\tau_{s} / \tau_{c}$ determines the particle structure, consistent with the literature. ${ }^{56,57}$ Specifically, the $D_{f}$ decreases from 3 at $\tau_{s} / \tau_{c} \approx 0.6-$ 0.8 down to $D_{f}=1.8$ within a narrow $\tau_{s} / \tau_{c}$ range (at $\tau_{s} / \tau_{c}=0.6-3$ ). So, when $\tau_{s}$ is about 3 times larger than $\tau_{c}$, the collision mechanism determines the particle structure, although sintering still takes place. The $D_{f m}$ starts dropping already from $\tau_{s} / \tau_{c} \approx 0.01$ and continues to decrease within a broader range of $\tau_{s} / \tau_{c}$ ratios than $D_{f}$. The ED-obtained $D_{f}$ is compared to the equilibrium $D_{f}$ of aggregates generated by MC assuming constant $\tau_{s} / \tau_{c}$ ratio $\left(\operatorname{circles}^{58}\right)$. Even though both the ED- and the MC-derived $D_{f}$ start dropping at $\tau_{s} / \tau_{c} \approx 0.6-0.9$, the ED-obtained $D_{f}$ decreases within a narrower range of $\tau_{s} / \tau_{c}$ ratios, indicating that aggregates quickly attain their asymptotic $D_{f}$ of $\sim 1.8$ once sintering is suppressed. In fact, the $D_{f}$ obtained ${ }^{58}$ at constant $\tau_{s} / \tau_{c}$ follows more closely the evolution of the ED-derived $D_{f m}$ at $\tau_{s} / \tau_{c}>2$. The time evolutions of $D_{f}$ and $D_{f m}$ is shown for aggregates with $r_{p 0}=1 \mathrm{~nm}$ in Fig. S5 of the Supplementary Information.

\section{Figure 7}

Figure 8 shows the fractal dimension, $D_{f}$, in the process parameter space of the reactor temperature, $T$, and the effective number of primary particles per aggregate/agglomerate, $n_{p}$ (corresponding e.g., to increasing height above the burner in flame stray pyrolysis reactors), for $r_{p, 0}=1 \mathrm{~nm}$. The white top-right corner of the color map corresponds to non-simulated conditions, as ED simulations become computationally expensive for large sintered aggregates. The isotherms of $D_{f}=2$ (broken line) and 2.5 (solid line) are also shown. Initially when aggregates are small consisting of $n_{p}<2$, they are perfect spheres at all temperatures. As nanoparticles grow by simultaneous sintering and agglomeration, they form compact aggregates that, upon further collisions, lead to fractal-like agglomerates consisting of aggregates. The aggregates attain their asymptotic structure of $\sim 1.8$ for $n_{p}>100$ at all temperatures investigated here, consistent with Jullien et al. ${ }^{59}$ These results are consistent with wide-angle light scattering measurements in hot wall reactors ${ }^{60}$ showing that the fractal-like morphology of silica aggregates is hardly affected at sintering temperatures below $1500 \mathrm{~K}$, exhibiting $D_{f} \approx 1.6-1.7$, while compact spherical-like particles with $D_{f}=2.5-2.8$ are formed at higher temperatures. The ED-derived $D_{f}$ are also compared to those obtained by 
SAXS measurements in flames (color-coded symbols based on the $D_{f}$ ) at $1665\left(\right.$ circle $\left.^{61}\right), 1950\left(\right.$ square $\left.^{62}\right)$ and $2000 \mathrm{~K}$ (triangle ${ }^{63}$ ), corresponding to $1.66,2.28$ and 2.5 , respectively, in qualitative agreement with the present simulations. It should be noted, however, that the actual number of primary particles per aggregate/agglomerate that is typically counted by transmission electron microscopy is expected to be smaller than the effective one shown here, especially at high temperatures, which is based on the initial size of the primary particles.

\section{Figure 8}

The overall collision frequency function is commonly used in PBE models for the process design of aerosol reactors. At the early stages of particle growth, all particles are monodisperse and the time between collisions is sufficiently long to fully coalesce. Thus, bigger spheres are formed that collide with coagulation rate that increases with increasing particle size. However, bigger primary particles lead to increased sintering time and formation of aggregates, if the collision and sintering times are of the same order of magnitude. This enhances the collision frequency significantly as they collide more frequently with other particles in the free molecular regime. Figure 9 shows the enhancement of the ED-obtained collision frequency function, $\beta$, over the collision kernel of Fuchs,

$\beta_{\text {Fuchs,agglo }}$ for monodisperse unsintered agglomerates, as function of the normalized aggregate/agglomerate mobility radius for aggregates made by primary particles (a) with $r_{p, 0}=5 \mathrm{~nm}$ at 1000 (black line), 1500 (blue line), 1750 (green line) and $2000 \mathrm{~K}$ (red line) and (b) with $r_{p, 0}=0.5$ (purple line), 1 (red line), 2 (green line) and $5 \mathrm{~nm}$ (blue line) at $2000 \mathrm{~K}$. Initially, the enhancement of the collision frequency over $\beta_{\text {Fuchs,agglo }}$ is close to 1 . For big initial primary particles $\left(r_{p, 0}=5 \mathrm{~nm}\right.$, Fig. 9a), the average collision frequency enhancement increases rapidly with increasing aggregate size reaching a plateau at $\sim 90 \%$ (horizontal broken line) once the aggregate $r_{m}$ doubles with respect to the initial primary particle size (i.e., at $r_{m} / r_{p, 0} \approx 2$ ), regardless of the process temperature (Fig. $9 \mathrm{a}$ ), so that:

$$
\beta_{p, a g g r}=1.90 \cdot \beta_{\text {Fuchs,agglo }}
$$

This enhancement of $\sim 90 \%$ is observed in the near free molecular-transition regime, since the $r_{m}$-based gas $K n$ number of aggregates with $r_{p, 0}=5 \mathrm{~nm}$ (Fig. 9a) is in the range of $2-85$ at all temperatures shown here. At the conditions discussed in Fig. 9a, sintering is very limited and practically aggregates consisting of primary particles with small sinter necks are formed. The collisional enhancement of $90 \%$ is attributed to the sintering-induced polydispersity of the constituent primary particles of the silica aggregated that is not accounted for in the Fuchs kernel of monodisperse agglomerates. The ED-predicted enhancement of the collision kernel is in excellent agreement with the enhancement of uncharged titania nanoparticles obtained by experiments in premixed flames. ${ }^{64}$ Equation 10 can be readily used in particle-fluid models for the simulation of industrial synthesis of fractal-like 
aggregates with primary particles of $r_{p, 0} \geq 5 \mathrm{~nm}$ grown by coagulation and sintering for a wide range of process temperatures $(1000-2000 \mathrm{~K})$.

At high temperature (Fig. 9b), the average enhancement is much lower during the particle growth investigated here for all $r_{p, 0}$ smaller than $5 \mathrm{~nm}$. This is attributed to the greater degree of sintering that leads to more compact aggregates, which tend to behave more like fully coalescing spheres than coagulating agglomerates. In fact, for very small $r_{p, 0}($ e.g., $0.5 \mathrm{~nm})$ the enhancement becomes smaller than unity as particles grow practically by full coalescence, following more closely the collision rate of spherical particles (Fig. S4b). The collisional enhancement of the ED-obtained silica aggregates over the Fuchs kernel of monodisperse spherical nanoparticles, $\beta_{F u c h s, s p h}$, is shown in the Supplementary Information (Fig. S4) for the conditions of Figure 9. The enhancement of the coagulation rate of fractal-like aggregates over $\beta_{F u c h s, s p h}$ is between $2-3.7$ (Supplementary Information: Fig. S4a), consistent with the experimentally-obtained collisional enhancement of flame-made titania nanoparticles calculated by particle size distribution measurements ${ }^{65}$ and the enhancement derived by molecular dynamics simulations accounting for dipole-dipole interactions ${ }^{66}$. In addition, the collisional enhancement over $\beta_{F u c h s, s p h}$ increases with increasing process temperature for $r_{p, 0}=5 \mathrm{~nm}$ (Fig. S4a), where the collision rate is bigger than the sintering rate, consistent with experiments ${ }^{65}$ and simulations ${ }^{66,67}$. At high temperatures and small initial sizes (e.g., Fig. S4b: e.g., for $\left.r_{p, 0}=0.5 \mathrm{~nm}\right)$, particles behave as fully-coalescing spheres early on $\left(r_{m} / r_{p, 0}=1-3\right)$, exhibiting an enhancement of $\sim 18 \%$ in the free molecular regime over the $\beta_{F u c h s, s p h}$ of monodisperse spheres, consistent with the literature ${ }^{68}$. As they grow further $\left(r_{m} / r_{p, 0}>3\right)$, the sintering rate decreases and non-spherical aggregates are formed that coagulate faster than spheres with equivalent size, leading in larger collisional enhancement. However, since these nonspherical aggregates are still compact (Fig. $7 \mathrm{a}: D_{f}>1.8$ for $r_{p, 0}=0.5 \mathrm{~nm}$ ), they exhibit a collision frequency function which is in-between the limits of perfect monodisperse spheres (Fig. S4b, $\beta / \beta_{\text {Fuchs,sph }}>1.18$ for $r_{p, 0}=0.5 \mathrm{~nm}$ ) and unsintered agglomerates (Fig. 9b, $\beta / \beta_{\text {Fuchs, agglo }}<1$ for $r_{p, 0}=0.5 \mathrm{~nm}$ ).

\section{Figure 9}

\section{Conclusions}

Coagulation of silica nanoparticles by concurrent agglomeration and viscous flow sintering is investigated by offlattice event-driven (ED) simulations at high temperatures from the free molecular to the transition regime, under dilute conditions. At low temperatures, coagulation by agglomeration is the dominant particle growth mechanism 
resulting in the formation of agglomerates consisting of primary particles in point contact. At this limit of pure agglomeration, particles reach a self-preserving size distribution with gyration-, mobility- and volume-based geometric standard deviations of 2.27, 2.03 and 1.41, respectively, consistent with literature. At high temperatures (i.e., $T>1500 \mathrm{~K}$ ), corresponding to the early stages of particle growth in flame reactors, sintering dominates, especially for small primary particles. In this particle growth region, the simultaneous agglomeration and sintering lead to narrower size distributions and delays the growth of fractal-like particles compared to growth by agglomeration in the absence of sintering. At all conditions investigated here, the volume-based geometric standard deviation attains a self-preserving value of 1.41 in the free molecular regime, regardless of the degree of aggregation. Ballistic cluster-cluster agglomeration with concurrent sintering leads to fractal-like particles with asymptotic fractal dimension, $D_{f} \approx 1.8$, consistent with experiments. However, when sintering prevails (i.e., at higher temperature), the attainment of the asymptotic $D_{f}$ is delayed. This delay indicates aggregate densification and reduction in the particle surface area which, in turn, affects catalytic or sensing properties of the end-product. The mass-mobility exponent, $D_{f m}$, is typically evolving more slowly than the $D_{f}$ and does not attain its asymptotic value when the sintering mechanism is significant. Nonetheless, the ratio of the characteristic sintering time to the characteristic collision time suffices to define the aggregate structure $\left(D_{f}\right)$, regardless of process temperature or initial primary particle size. Therefore, the dimensionless time can be used as a design parameter to produce particles with desired structure $\left(D_{f}\right)$, which is related to the nanomaterial optical properties.

The collision frequency function of aggregates coagulating by agglomeration and viscous flow sintering is also obtained by ED simulations and its enhancement over the theoretical coagulation rate of Fuchs for monodisperse agglomerates is determined. For compact aggregates that grow mainly by sintering the enhancement of their collision frequency function over the collision kernel of Fuchs is less than 30\%, as they behave as fully coalescing spheres. However, for aggregates that grow primarily by agglomeration and little or no sintering, collision frequency enhancement is about $90 \%$, consistent with experiments. The proposed simple-to-use collision kernel is obtained without making a priori assumptions about particle structure and accounts for the sinteringinduced primary particle polydispersity as well as aggregate polydispersity. This collision kernel equation along with the simple relationship that quantifies the evolution of particle morphology with the dimensionless characteristic time can be readily employed in particle dynamics simulations (e.g., population balance models) coupled with computational fluid dynamics models to improve the design of aerosol processes at industrial scales. 


\section{Conflict of Interest}

The authors declare no conflict of interest.

\section{Acknowledgements}

Financial support from The Melbourne School of Engineering of The University of Melbourne, Australia is gratefully acknowledged.

\section{Supporting Information}

Validation of the simulations based on the collision frequency function and geometric standard deviation of fully coalescing spheres, comparison of the simulated agglomerate size and geometric standard deviation with experiments, and time evolutions of particle structure descriptors are shown in the Supplementary Information. 


\section{References}

1. Ulrich G. D. (1984). Chem Eng News 62 (32) 22-29.

2. Hanker, J. S., \& Giammara, B. L. (1988). Biomaterials and biomedical devices. Science, 242(4880), 885-892.

3. Mädler, L., Krumeich, F., Burtscher, P., \& Moszner, N. (2006). Visibly transparent \& radiopaque inorganic organic composites from flame-made mixed-oxide fillers. Journal of Nanoparticle Research, 8(3-4), 323-333.

4. Bettini, L. G., Diamanti, M. V., Sansotera, M., Pedeferri, M. P., Navarrini, W., \& Milani, P. (2016). Immobilized $\mathrm{TiO} 2$ nanoparticles produced by flame spray for photocatalytic water remediation. Journal of Nanoparticle Research, 18(8), 238.

5. Kho, Y. K., Teoh, W. Y., Iwase, A., Mädler, L., Kudo, A., \& Amal, R. (2011). Flame preparation of visible-lightresponsive BiVO4 oxygen evolution photocatalysts with subsequent activation via aqueous route. ACS applied materials \& interfaces, 3(6), 1997-2004.

6. Guild, C., Biswas, S., Meng, Y., Jafari, T., Gaffney, A. M., \& Suib, S. L. (2014). Perspectives of spray pyrolysis for facile synthesis of catalysts and thin films: An introduction and summary of recent directions. Catalysis Today, 238, 87-94.

7. Henning, D. F., Merkl, P., Yun, C., Iovino, F., Xie, L., Mouzourakis, E., Moularas, C., Deligiannakis, Y., Henriques-Normark, B., Leifer, K., \& Sotiriou, G. A. (2019). Luminescent CeO2: Eu3+ nanocrystals for robust in situ $\mathrm{H} 2 \mathrm{O} 2$ real-time detection in bacterial cell cultures. Biosensors and Bioelectronics, 132, $286-293$.

8. Yang, L., Ren, Y., Pan, W., Yu, Z., Tong, L., Li, N., \& Tang, B. (2016). Fluorescent nanocomposite for visualizing cross-talk between microRNA-21 and hydrogen peroxide in ischemia-reperfusion injury in live cells and in vivo. Analytical chemistry, 88(23), 11886-11891.

9. Joshi, R. K., \& Kruis, F. E. (2006). Influence of Ag particle size on ethanol sensing of Sn O 1.8: Ag nanoparticle films: A method to develop parts per billion level gas sensors. Applied physics letters, 89(15), 153116.

10. Tran-Phu, T., Daiyan, R., Fusco, Z., Ma, Z., Amal, R., \& Tricoli, A. (2020). Nanostructured $\beta$-Bi2O3 Fractals on Carbon Fibers for Highly Selective CO2 Electroreduction to Formate. Advanced Functional Materials, 30(3), 1906478.

11. Ulrich, G. D. (1971). Theory of particle formation and growth in oxide synthesis flames. Combustion Science and Technology, 4(1), 47-57.

12. Goudeli, E. (2019). Nanoparticle growth, coalescence, and phase change in the gas-phase by molecular dynamics. Current Opinion in Chemical Engineering, 23, 155-163.

13. Mountain, R. D., Mulholland, G. W., \& Baum, H. (1986). Simulation of aerosol agglomeration in the free molecular and continuum flow regimes. Journal of Colloid and Interface Science, 114(1), 67-81.

14. Oh, C., \& Sorensen, C. M. (1997). Light scattering study of fractal cluster aggregation near the free molecular regime. Journal of Aerosol Science, 28(6), 937-957.

15. Goudeli, E., Eggersdorfer, M. L., \& Pratsinis, S. E. (2015). Aggregate characteristics accounting for the evolving fractal-like structure during coagulation and sintering. Journal of Aerosol Science, 89, 58-68.

16. Mulholland, G. W., Samson, R. J., Mountain, R. D., \& Ernst, M. H. (1988). Cluster size distribution for free molecular agglomeration. Energy \& Fuels, 2(4), 481-486.

17. Zurita-Gotor, M., \& Rosner, D. E. (2002). Effective diameters for collisions of fractal-like aggregates: Recommendations for improved aerosol coagulation frequency predictions. Journal of colloid and interface science, 255(1), 10-26.

18. Thajudeen, T., Gopalakrishnan, R., \& Hogan Jr, C. J. (2012). The collision rate of nonspherical particles and aggregates for all diffusive Knudsen numbers. Aerosol Science and Technology, 46(11), 1174-1186.

19. Goudeli, E., Eggersdorfer, M. L., \& Pratsinis, S. E. (2015). Coagulation-Agglomeration of fractal-like particles: Structure and self-preserving size distribution. Langmuir, 31(4), 1320-1327.

20. Goudeli, E., Eggersdorfer, M. L., \& Pratsinis, S. E. (2016). Coagulation of agglomerates consisting of polydisperse primary particles. Langmuir, 32(36), 9276-9285.

21. Kelesidis, G. A., Goudeli, E., \& Pratsinis, S. E. (2017). Flame synthesis of functional nanostructured materials and devices: Surface growth and aggregation. Proceedings of the Combustion Institute, 36(1), 29-50.

22. Artelt, C., Schmid, H. J., \& Peukert, W. (2003). On the relevance of accounting for the evolution of the fractal dimension in aerosol process simulations. Journal of Aerosol Science, 34(5), 511-534.

23. Al Zaitone, B., Schmid, H. J., \& Peukert, W. (2009). Simulation of structure and mobility of aggregates formed by simultaneous coagulation, sintering and surface growth. Journal of aerosol science, 40(11), 950-964.

24. Sander, M., West, R. H., Celnik, M. S., \& Kraft, M. (2009). A detailed model for the sintering of polydispersed nanoparticle agglomerates. Aerosol Science and Technology, 43(10), 978-989. 
25. Seto, T., Hirota, A., Fujimoto, T., Shimada, M., \& Okuyama, K. (1997). Sintering of polydisperse nanometersized agglomerates. Aerosol Science and Technology, 27(3), 422-438.

26. Mandelbrot, B. B. (1982). The Fractal Geometry of. Nature, 394-397.

27. Park, K., Cao, F., Kittelson, D. B., \& McMurry, P. H. (2003). Relationship between particle mass and mobility for diesel exhaust particles. Environmental science \& technology, 37(3), 577-583.

28. Meakin, P., Donn, B., \& Mulholland, G. W. (1989). Collisions between point masses and fractal aggregates. Langmuir, 5(2), 510-518.

29. Fuchs, N.A., The mechanics of aerosols. Dover Publications Inc.: New York, 1964.

30. Vincenti, W. G., Kruger, C. H. Introduction to Physical Gas Dynamics; Krieger: Malabar, FL, 1965.

31. Pierce, F., Sorensen, C. M., \& Chakrabarti, A. (2006). Computer simulation of diffusion-limited cluster-cluster aggregation with an Epstein drag force. Physical Review E, 74(2), 021411.

32. Goudeli, E., Lee, J., \& Hogan Jr, C. J. (2020). Silica nanocluster binding rate coefficients from molecular dynamics trajectory calculations. Journal of Aerosol Science, 105558.

33. Nietiadi, M. L., Umstätter, P., Tjong, T., Rosandi, Y., Millán, E. N., Bringa, E. M., \& Urbassek, H. M. (2017). The bouncing threshold in silica nanograin collisions. Physical Chemistry Chemical Physics, 19(25), 1655516562.

34. D’Alessio, A., Barone, A. C., Cau, R., D’Anna, A., \& Minutolo, P. (2005). Surface deposition and coagulation efficiency of combustion generated nanoparticles in the size range from 1 to $10 \mathrm{~nm}$. Proceedings of the Combustion Institute, 30(2), 2595-2603.

35. Nietiadi, M. L., Rosandi, Y., \& Urbassek, H. M. (2020). Bouncing of Hydroxylated Silica Nanoparticles: an Atomistic Study Based on REAX Potentials. Nanoscale Research Letters, 15, 1-6.

36. Ennis, B. J., Tardos, G., \& Pfeffer, R. (1991). A microlevel-based characterization of granulation phenomena. Powder Technology, 65(1-3), 257-272.

37. Heine, M. C., \& Pratsinis, S. E. (2007). Brownian coagulation at high concentration. Langmuir, 23(19), 98829890.

38. S.K. Friedlander, Smoke, Dust, and Haze: Fundamentals of Aerosol Dynamics. $2^{\text {nd }}$ ed., Oxford University Press, New York, 2000.

39. Koch, W., \& Friedlander, S. K. (1990). The effect of particle coalescence on the surface area of a coagulating aerosol. Journal of Colloid and Interface Science, 140, 419-427.

40. M.P. Allen, D.J. Tildesley, Computer Simulation of Liquids. Oxford University Press, New York, 2000.

41. Kelkar, A. V., Dong, J., Franses, E. I., \& Corti, D. S. (2013). New models and predictions for Brownian coagulation of non-interacting spheres. Journal of colloid and interface science, 389(1), 188-198.

42. Kingery, W. D., Bowen, H. K., \& Uhlmann, D. R. (1976). Introduction to ceramics. Wiley: New York.

43. Tsantilis, S., Briesen, H., \& Pratsinis, S. E. (2001). Sintering Time for Silica Particle Growth. Aerosol Science and Technology, 34, 237-246.

44. Kirchhof, M. J., Forster, H., Schmid, H-J., \& Peukert, W. (2012). Sintering kinetics and mechanism of virteous nanoparticles. Journal of Aerosol Science, 45, 26-39.

45. Frenkel, J. J. (1945). Viscous flow of crystalline bodies under the action of surface tension. J. Phys., 9, 385.

46. Cazals, F., Kanhere, H., \& Loriot, S. (2011). Computing the volume of a union of balls: a certified algorithm. ACM Transactions on Mathematical Software (TOMS), 38(1), 1-20.

47. Reinhold, A., \& Briesen, H. (2012). Numerical behavior of a multiscale aggregation model—coupling population balances and discrete element models. Chemical engineering science, 70, 165-175.

48. Lai, F. S., Friedlander, S. K., Pich, J., \& Hidy, G. M. (1972). The self-preserving particle size distribution for Brownian coagulation in the free-molecule regime. Journal of Colloid and Interface Science, 39(2), 395-405.

49. Landgrebe, J. D., \& Pratsinis, S. E. (1989). Gas-phase manufacture of particulates: interplay of chemical reaction and aerosol coagulation in the free-molecular regime. Industrial \& engineering chemistry research, 28(10), 1474-1481.

50. Eggersdorfer, M. L., Kadau, D., Herrmann, H. J., \& Pratsinis, S. E. (2011). Multiparticle sintering dynamics: from fractal-like aggregates to compact structures. Langmuir, 27(10), 6358-6367.

51. Kelesidis, G. A., Furrer, F. M., Wegner, K., \& Pratsinis, S. E. (2018). Impact of humidity on silica nanoparticle agglomerate morphology and size distribution. Langmuir, 34(29), 8532-8541.

52. Meierhofer, F., Mädler, L., \& Fritsching, U. (2020). Nanoparticle evolution in flame spray pyrolysis—Process design via experimental and computational analysis. AIChE Journal, 66(2), e16885.

53. Camenzind, A., Schulz, H., Teleki, A., Beaucage, G., Narayanan, T., \& Pratsinis, S. E. (2008). Nanostructure evolution: from aggregated to spherical $\mathrm{SiO} 2$ particles made in diffusion flames. European Journal of Inorganic Chemistry, 2008(6), 911-918. 
54. Goudeli, E., Gröhn, A. J., \& Pratsinis, S. E. (2016). Sampling and dilution of nanoparticles at high temperature. Aerosol Science and Technology, 50(6), 591-604.

55. Meakin, P., \& Jullien, R. (1988). The effects of restructuring on the geometry of clusters formed by diffusionlimited, ballistic, and reaction-limited cluster-cluster aggregation. The Journal of chemical physics, 89(1), 246250.

56. Schmid, H-J., Tejwani, S., Artelt, C., \& Peukert, W. (2004). Monte Carlo simulation of aggregate morphology for simultaneous coagulation and sintering. Journal of Nanoparticle Research, 6, 613-626.

57. Hawa, T., \& Zachariah, M. R. (2007). Development of a phenomenological scaling law for fractal aggregate sintering from molecular dynamics simulation. Journal of Aerosol Science, 38, 793-806.

58. Schmid, H. J., Al-Zaitone, B., Artelt, C., \& Peukert, W. (2006). Evolution of the fractal dimension for simultaneous coagulation and sintering. Chemical Engineering Science, 61(1), 293-305.

59. Jullien, R., Kolb, M., \& Botet, R. (1984). Diffusion limited aggregation with directed and anisotropic diffusion. Journal de Physique, 45, 395-399.

60. Babick, F., Hillemann, L., Stintz, M., Dillenburger, T., Pitz, M., Hellmann, A., Sergiy Antonyuk, Ripperger, S., Huber, F. J. T., Will, S., Wernet, R., Seipenbusch, M., Gensch, M., Weber, A., Kiesler, D., Kruis, E., Friehmelt, R., Sachweh, B. (2018). Multiparameter characterization of aerosols. Chemie Ingenieur Technik, 90(7), 923936.

61. Kammler, H. K., Beaucage, G., Mueller, R., \& Pratsinis, S. E. (2004). Structure of flame-made silica nanoparticles by ultra-small-angle X-ray scattering. Langmuir, 20(5), 1915-1921.

62. Rai, D. K., Beaucage, G., Vogtt, K., Ilavsky, J., \& Kammler, H. K. (2018). In situ study of aggregate topology during growth of pyrolytic silica. Journal of Aerosol Science, 118, 34-44.

63. Kammler, H. K., Beaucage, G., Kohls, D. J., Agashe, N., \& Ilavsky, J. (2005). Monitoring simultaneously the growth of nanoparticles and aggregates by in situ ultra-small-angle x-ray scattering. Journal of applied physics, 97(5), 054309.

64. Katzer, M., Weber, A. P., \& Kasper, G. (2001). Collision kinetics and electrostatic dispersion of airborne submicrometer fractal agglomerates. Journal of colloid and interface science, 240(1), 67-77.

65. Sharma, G., Dhawan, S., Reed, N., Chakrabarty, R., \& Biswas, P. (2019). Collisional growth rate and correction factor for TiO2 nanoparticles at high temperatures in free molecular regime. Journal of Aerosol Science, 127, 27-37.

66. Zhang, Y., Li, S., Yan, W., Yao, Q., \& Tse, S. D. (2011). Role of dipole-dipole interaction on enhancing Brownian coagulation of charge-neutral nanoparticles in the free molecular regime. The Journal of chemical physics, 134(8), 084501.

67. Ouyang, H., Gopalakrishnan, R., \& Hogan Jr, C. J. (2012). Nanoparticle collisions in the gas phase in the presence of singular contact potentials. The Journal of chemical physics, 137(6), 064316.

68. Buesser, B., Heine, M. C., \& Pratsinis, S. E. (2009). Coagulation of highly concentrated aerosols. Journal of Aerosol Science, 40(2), 89-100. 


\section{University Library}

\section{- M M I E E R VA A gateway to Melbourne's research publications}

Minerva Access is the Institutional Repository of The University of Melbourne

Author/s:

Eggersdorfer, ML;Goudeli, E

Title:

Structure and dynamics of fractal-like particles made by agglomeration and sintering

Date:

2020-12

Citation:

Eggersdorfer, M. L. \& Goudeli, E. (2020). Structure and dynamics of fractal-like particles made by agglomeration and sintering. AlChE Journal, 66 (12), https://doi.org/10.1002/ aic.17099.

Persistent Link:

http://hdl.handle.net/11343/276552 